International Journal of Engineering \& Technology, $7(2.7)(2018) 665-670$
International Journal of Engineering \& Technology
SPC
Website: www.sciencepubco.com/index.php/IJET
Research Paper

\title{
Automatic segmentation of multiple lesions in ultrasound breast image
}

\author{
Chelladurai . R ${ }^{1 *}$, Selvakumar. ${ }^{2}$, S. Poonguzhali ${ }^{3}$ \\ ${ }^{1,2}$ Assistant Professor Department of Electronics and Communication Engineering, \\ Koneru Lakshmaiah Education Foundation, Guntur, Andhra Pradesh, India \\ ${ }^{3}$ Professor Center for Medical Electronics, College of Engineering Guindy, Anna University, Chennai, India \\ *Email: chellad_blitz@yahoo.com
}

\begin{abstract}
Breast cancer is one of the leading cancer that affects woman all around the world. Nowadays ultra sound imaging technique is used to diagnose various cancer because of its non-ionizing, on-invasive, and cheap cost. Breast lesion region in ultrasound images are classified depending upon the contour, shape, size and textural features of the segmented region. Seed point is the initial step in segmentation of lesion regions and if that point is located outside the lesion region, it leads to wrong segmentation which results in misclassification of the lesion regions. To avoid this, most of the time the seed point is located manually. In order to avoid this manual intervention, we are proposing a novel method in locating the seed point and also segmenting the breast lesion region automatically. In this method, the image is processed with tan function for effective distinguishing of breast lesion and normal region. Then using the trained neural network, the seed point is automatically located inside the lesion region and from the seed point the region of the lesion is grown and segmented automatically. Most of the past works on automatic segmentation of lesion had concentrated only in single lesion region, but using this proposed method, we were able to automatically segment multiple lesion regions in the image. Outcome of the proposed method is to detect automatically and dynamically separate the lesion region in the range between $90 \%$ to $97.5 \%$ of images.
\end{abstract}

Keywords: Keywords: BPN (Back Propagated Network); Breast lesion ;Seed point; Tan Processing; Ultrasound (US) ;

\section{Introduction}

Ultrasound imaging is based on the transmission and receiving of sound waves above upper range of human hearing, now a days this technique is used to detect the various cancer because of it non ironizing, non-invasive and cheap cost when compare to other imaging like CT scanners, but the major drawback is poor quality of the image due to the present of speckle noise, due to this problem automatic segmentation of lesion region is very difficult. Automatic segmentation is start form a seed points. A seed points is a points inside the lesion region from which the segmentation is done using region growing method. If this point is located in the normal region the entire segmentation will be wrong, so in order to avoid this most of the time the seed point is located manually. A new method has been proposed to avoid the manual intervention and automatically locate the seed the points and also automatically segment the multiple lesions in the ultrasound image using tan function, textural features of the normal and lesion region and also by neural networks.

\section{Proposed methodology}

The US breast image is pre-processed using filter and tan function for effective distinguishes between normal and lesion regions. From the pre-processed image, the features are extracted from the normal and lesion regions and using these features the BPN network is trained. After training the network, the image is feed for the testing and automatically segmented. The block diagram for automatic segmentation is shown in Figure. 1

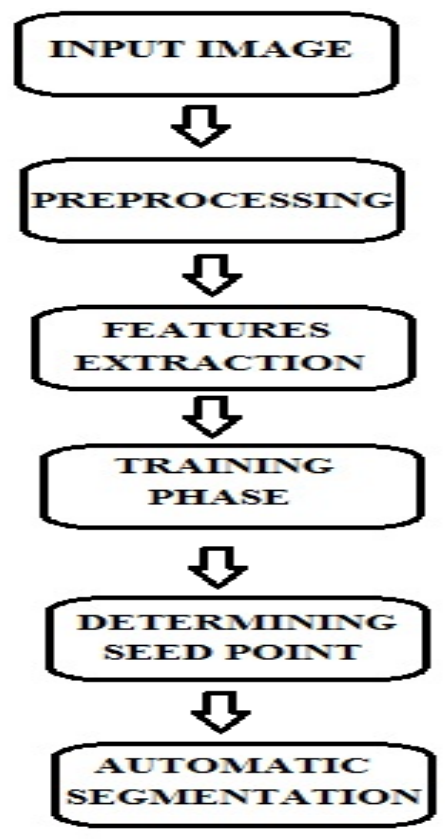

Fig. 1: Block diagram of automatic segmentation 


\subsection{Filtering the image}

US image is mostly degraded by the inherently existing speckle noise. Speckle noise is a multiplicative noise, which is directly proportional to the pixel intensity value. The degraded image affected by the speckle noise can be expressed as $\quad \mathrm{g}(\mathrm{x}, \mathrm{y})=\mathrm{I}(\mathrm{x}, \mathrm{y}) * \mathrm{n}(\mathrm{x}, \mathrm{y})$, $\mathrm{g}(\mathrm{x}, \mathrm{y})$ is the degraded image, $\mathrm{f}(\mathrm{x}, \mathrm{y})$ is the uncorrupted image and $\mathrm{n}(\mathrm{x}, \mathrm{y})$ is the noise multiplied with the image .

\subsubsection{Max filter}

Max filter is used for smoothing of the images by taking the max value in that particular masked region. Thereby, reducing the pixel intensity difference between the neighboring pixels. This filter is mainly used for pepper noise reduction and can be expressed as

$I(x, y)=\left(\begin{array}{c}\max \\ s, t \varepsilon s_{x y}\end{array}\right)\{g(s, t)\}$

where Sxy is the set of co-ordinate in a rectangular sub image window of size $(m * n)$ centered at $(x, y)$.

\subsubsection{Min filter}

Min filter retains the darkest value by performing the min operation. This filter is mainly used for removing salt noise. The expression is given by

$$
I(x, y)=\left(\begin{array}{c}
\min \\
s, t \varepsilon s_{x y}
\end{array}\right)\{g(s, t)\}
$$

\subsubsection{Geometric mean filter}

The Geometric filter attenuates he noise by lowering the variance. The geometric mean is given by

$I(x, y)=\left(\sum_{\varepsilon s_{x y}}^{s, t} g[s, t]^{1 / m n}\right)$

\subsubsection{Mean filter}

Mean filter replaces $\mathrm{I}(\mathrm{x}, \mathrm{y})$ value by the mean in the given classical window Sxy. Mean filter removes both salt and pepper noise. The expression is given by

$I(x, y)=\left(\begin{array}{c}\text { mean } \\ s, t \varepsilon s_{x y}\end{array}\right)\{g(s, t)\}$

\subsubsection{Median filter}

Median filter replaces I(x,y) value by taking the median in the given classical window Sxy. The median filter is given by

$I(x, y)=\left(\begin{array}{l}\text { median } \\ s, t \varepsilon s_{x y}\end{array}\right)\{g(s, t)\}$

\subsubsection{Wiener filter}

Wiener filter is mainly used for the removal of additive noise with the expression for the wiener filter is given by

$$
\mathrm{f}(\mathrm{x}, \mathrm{y})=\underset{\left(\mathrm{s}, \mathrm{t} \varepsilon \mathrm{S}_{\mathrm{xy}}\right)}{\text { wiener }}\{\mathrm{g}(\mathrm{s}, \mathrm{t})\}
$$

\subsubsection{Log combined wiener filter}

Speckle noise is a multiplicative noise which varies accordingly to the intensity value of the pixel. In this filter, initially log of the image is taken. Due to this, the multiplicative noise become additive and then wiener filter is applied to the log image so that the additive noise is removed. Then inverse log is obtained to reduce speckle noise and other noise in the image.

$f(x, y)=\exp ($ wiener $(\log (g(s, t)))) \quad(s, t) € S_{x y}$

\subsection{Contrast enhancement}

The filtered image is contrast enhanced using tan function. Here the region below half the maximum value is contrast enhanced and not the entire region. This is done because, most of the lesion lies below half of the maximum value of the image. The expression is given by

$\mathrm{c}(\mathrm{x}, \mathrm{y})=\tan (\mathrm{f}(\mathrm{x}, \mathrm{y}) *(\mathrm{pi} / 2))$

where $\mathrm{c}(\mathrm{x}, \mathrm{y})$ is the contrast enhanced image.

\subsection{Feature Extraction}

The statistical features like mean, standard deviation (SD) and entropy is found for both the normal and lesion regions. Since most of the lesion region have low mean when compared to normal region, so we are using mean as one of the features. Similarly, most of the breast lesion regions are more homogenous when compared to normal region, so we are extracting entropy and SD. In this project, we have extracted features from 5 images and then they are used for training the BPN network.

\subsection{Network Training}

Using the BPN network, the extracted features and the corresponding output is trained. BPN network is the widely used feed forward network to obtain the correct output from the given input features. Schematic diagram of the 3 inputs, 5 hidden layer and one output BPN network is shown in the Figure 2. In this network, the error between the output and the target is back propagated so that the network will be trained properly with less error. Here the input features are feed to the hidden layer after been multiplied with the weight associated with the hidden layer and input. The hidden layer function can be a log sigmoid function or any other transfer function. This output is feed as input to the output layer after been multiplied by the associated weight. The error between targeted value and the BPN network output is found. If it is less than the expected error, the error is back propagated. Depending upon the error, the associated weights are adjusted. The process is repeated until we get the expected minimum error. After reaching the desired error, the process is stop. Atlas a new network (net) is obtained. In this project, after extracting the features, the BPN network is trained and a new network is obtained

\subsection{Determining the Seed Point}

In this step, features like mean, entropy and SD are obtained from the testing image with the dimension of $18 * 18$ pixels. The obtained features are feed as input to the trained network. If the inputs are similar to lesion features, then a corresponding output of 1 is produced. Similarly, if it is similar to normal region, output of 0 is produced. Some of the boundary connected normal regions in US images have similar features compared to lesion region. These regions must be deleted. Careful observation was made on 40 tan processed images. In all these images, the lesion regions are surrounded by high pixel intensity when compared to the normal region. Depending upon this observation, the regions are evaluated. Then, the seed point is found within the lesion region. 


\subsection{Automatic Segmentation}

The region growing method is applied to automatically grow the lesion region surrounding the seed point. After the completion of the region growing process, the boundary of the lesion region is found using the edge detection method in the testing image.

IPUT TO HIDDEN MDDENTOOCTPT

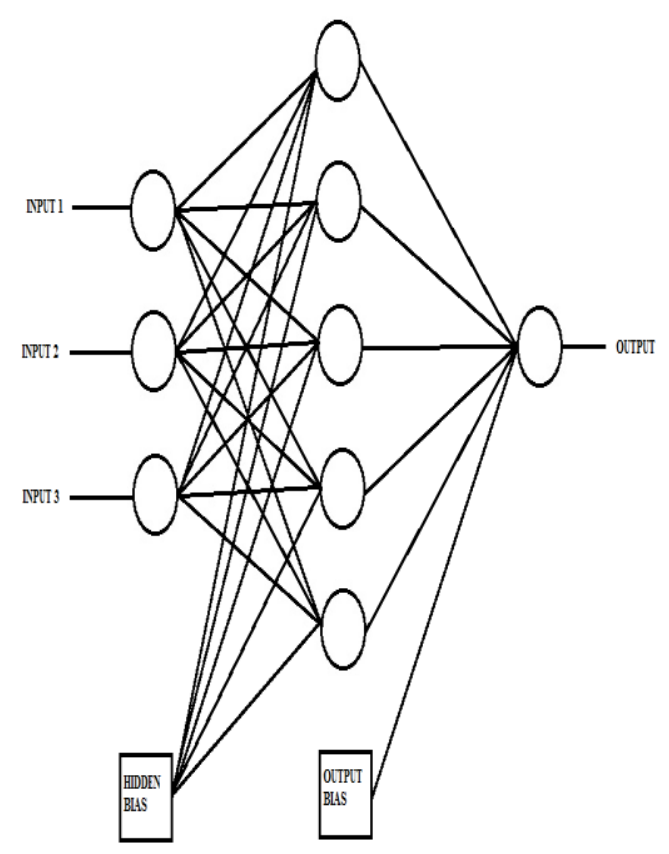

Fig. 2: Schematic diagram of BPN network

\section{Results}

\subsection{Evaluating the Filters}

To evaluate the filters, first a standard image is obtained and speckle noise is added with the image with mean of 0 and with different variance. Speckle noise is a multiplicative noise which is inherently present in the image. After adding the speckle noise, the image is filtered using the various filters as shown in Table 1. Finally, peaksignal to noise ratio (PSNR) is calculated between the standard image without noise and with various filtered image as shown in the Table 1. Depending upon the PSNR value, the best filter is found to be wiener filter and this filter is used in the program.

Table 1: Filter results

\begin{tabular}{|l|l|l|}
\multicolumn{2}{c}{ Table 1: Filter results } \\
\hline & $\begin{array}{l}\text { Ultrasound breast im- } \\
\text { age with variance=0.04 } \\
\text { (PSNR) }\end{array}$ & $\begin{array}{l}\text { Ultrasound breast } \\
\text { image with vari- } \\
\text { ance=0.02 (PSNR) }\end{array}$ \\
\hline Max & 13.78 & 15.36 \\
\hline Min & 14.67 & 16.22 \\
\hline Gem-Mean & 23.24 & 24.10 \\
\hline Mean & 24.06 & 24.95 \\
\hline Median & 22.84 & 24.53 \\
\hline Wiener & 25.45 & 27.91 \\
\hline $\begin{array}{l}\text { Wiener with } \\
\text { Log }\end{array}$ & 27.70 & 27.53 \\
\hline
\end{tabular}

\subsection{Processing With Tan Function}

For effective distinguishing of the lesion region from the normal region, every pixel value of the input image is processed with tan function. The input image and its histogram are shown in the Figure 3 and Figure 4 . And, the tan processed image and its histogram is shown in the Figure 5 and Figure 6.

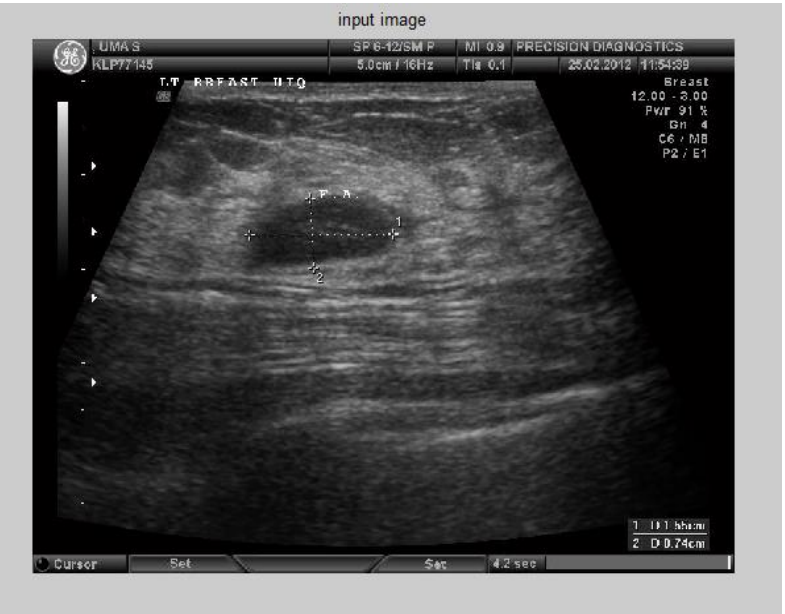

Fig. 3: Input image

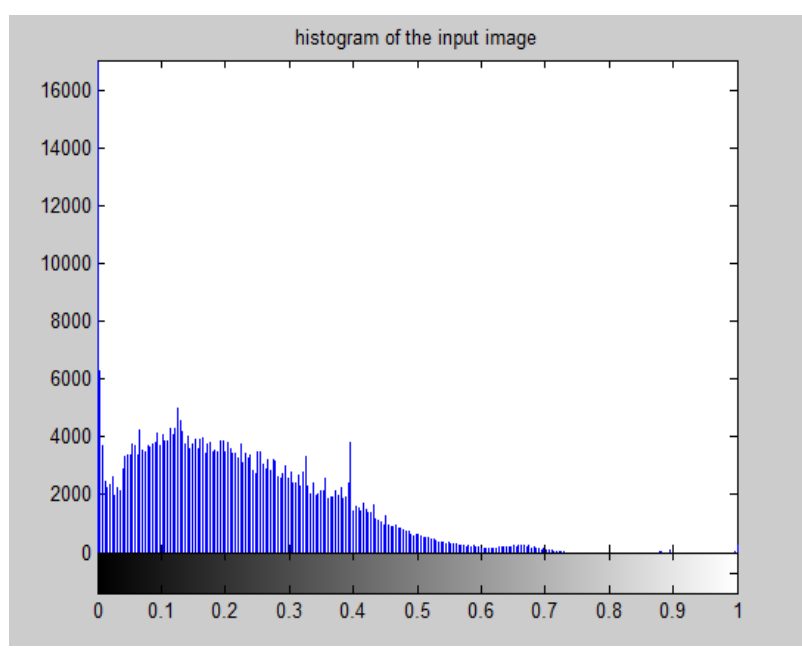

Fig.4: Histogram of the input image

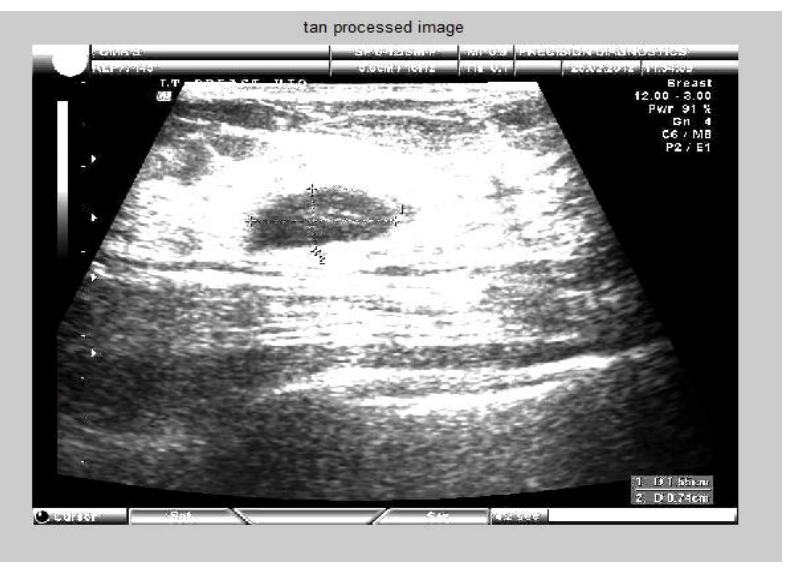

Fig.5: Tan processed image

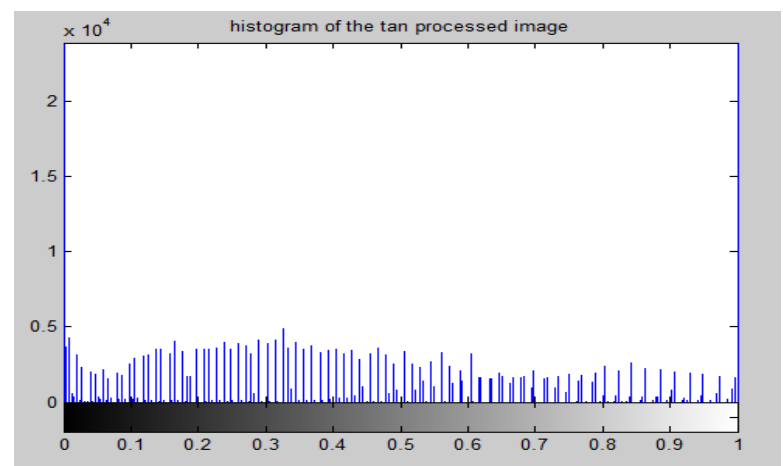

Fig.6: Histogram of tan processed image 


\subsection{Extracting the Features}

The features like mean, SD and entropy for lesion region and normal region is extracted for 5 different images. In Table 2, the extracted features are shown for normal region and in Table 3 the extracted features are shown for lesion region for all the 5 images.

\begin{tabular}{|c|c|c|}
\multicolumn{3}{|c}{ Table 2: Normal region features } \\
\hline Mean & Entropy & Standard Deviation \\
\hline $\mathbf{0 . 7 0 9 3}$ & 5.87 & 0.0320 \\
\hline $\mathbf{0 . 4 1 8 2}$ & 4.16 & 0.00062 \\
\hline $\mathbf{0 . 1 6 0 4}$ & 4.92 & 0.017 \\
\hline $\mathbf{0 . 5 5 4 9}$ & 5.80 & 0.0504 \\
\hline $\mathbf{0 . 3 5 5 9}$ & 4.92 & 0.0086 \\
\hline
\end{tabular}

Table 3: Breast lesion features

\begin{tabular}{|c|c|c|}
\hline Mean & Entropy & Standard Deviation \\
\hline $\mathbf{0 . 0 3 6 4}$ & 4.33 & 0.0039 \\
\hline $\mathbf{0 . 1 1 2 6}$ & 4.83 & 0.0097 \\
\hline $\mathbf{0 . 3 1 0 4}$ & 4.06 & 0.0050 \\
\hline $\mathbf{0 . 1 3 9 7}$ & 4.83 & 0.0133 \\
\hline $\mathbf{0 . 3 1 9 7}$ & 4.92 & 0.0070 \\
\hline
\end{tabular}

\subsection{Training Phase}

The extracted features from the training images are feed as input to the BPN network with the associated output. The transfer function used here is logsigmoid function. Here the maximum no of epoch is set to 10000 with goal of .0001 mean square error between the obtained output and the targeted output. The algorithm used is levenberg-Marquardt algorithm. The training process of the neural network is shown the Figure 7. The input and the output of the trained network are shown in the Figure 8.

\subsection{Testing Phase}

From every $18 * 18$ pixel of the testing image, the features are found and fed as the input to the newly trained BPN network. From the BPN outputs, the seed points are found inside the lesion regions. Using the region growing method, the lesion regions are automatically segmented from the seed points. Figure $(9,13)$ shows the input image and Figure $(10,14)$ tan processed image. In tan processing, every pixel of the input image is tan processed for effective distinguishing of the lesion region from the normal region. Figure $(11,15)$ shows the automatically located seed point inside the lesion region and finally the lesion region is automatically segmented using the region growing method as shown in the Figure $(12,16)$.
A Neural Network Training (nntraintool)

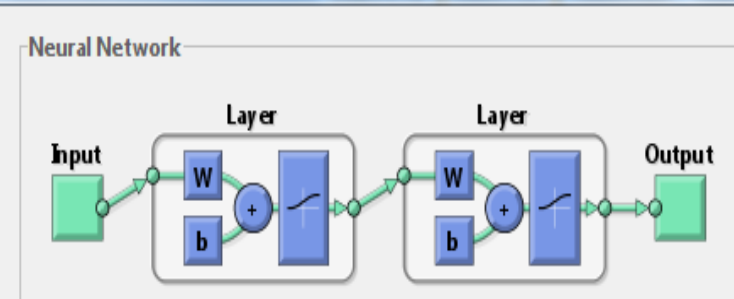

$\begin{array}{ll}\text { Algorithms } & \\ \text { Training: } & \text { Levenberg-Marquardt (train } / m \text { ) } \\ \text { Performance: } & \text { Mean Squared Error (mse) }\end{array}$

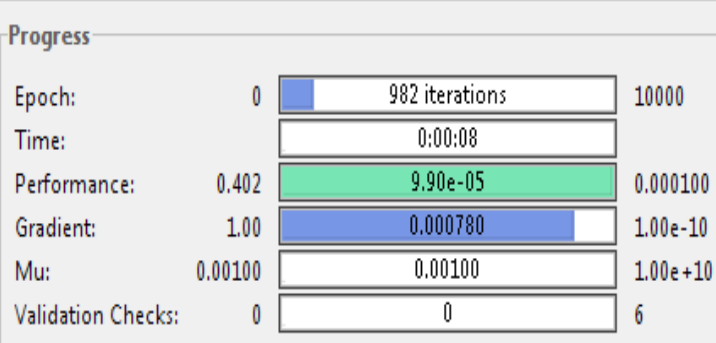

Plots

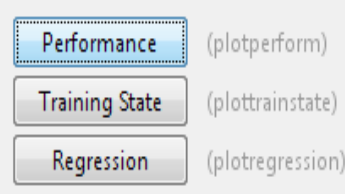

Plot Interval: 1 1epochs

Performance goal met.

Stop Training

Cancel

Fig.7: Neural network training process

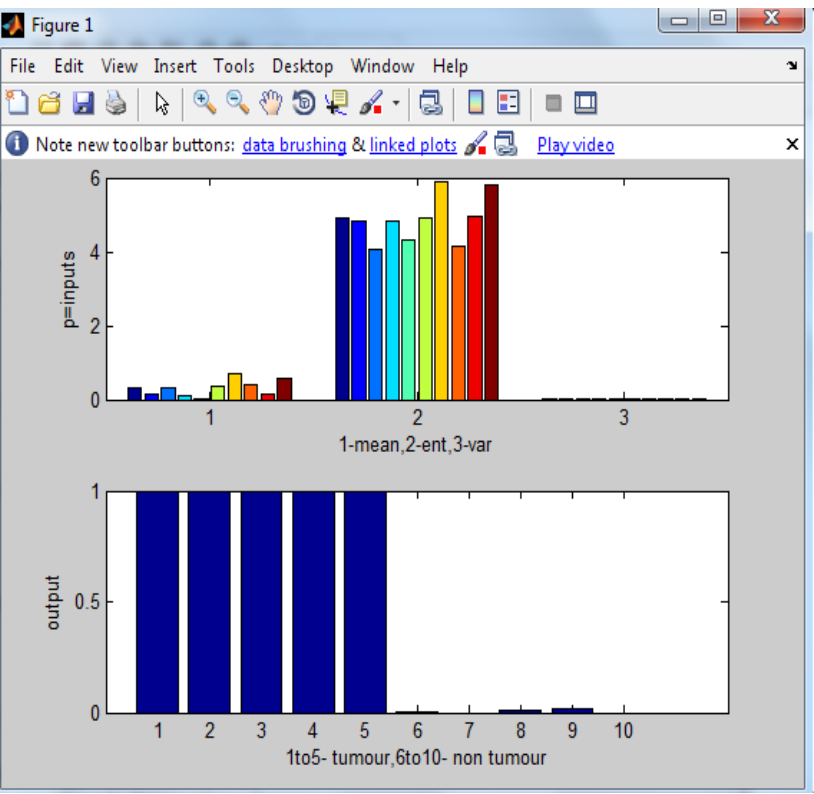

Fig.8: Input and output of the trained neural network 


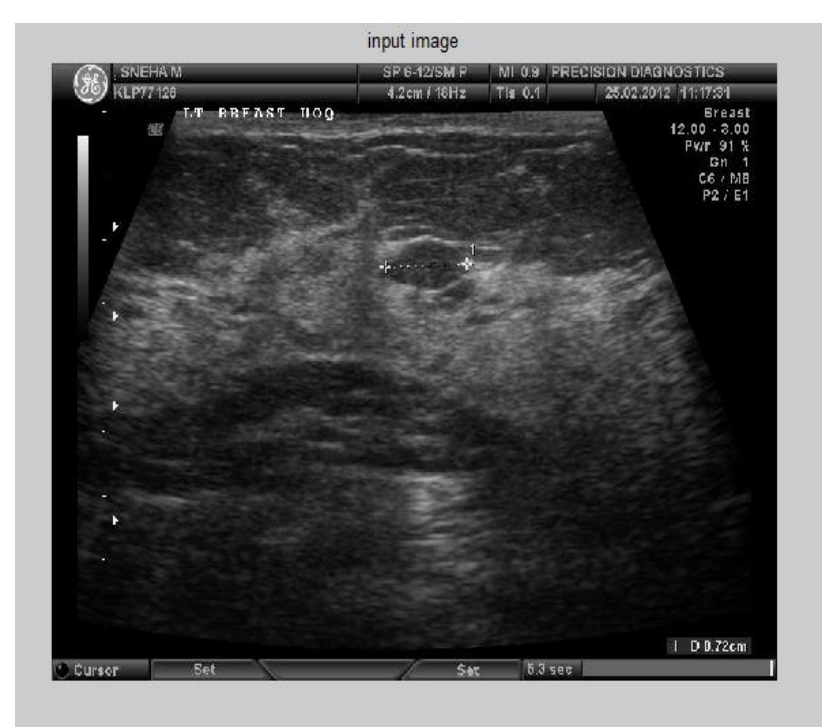

Fig.9: Input image

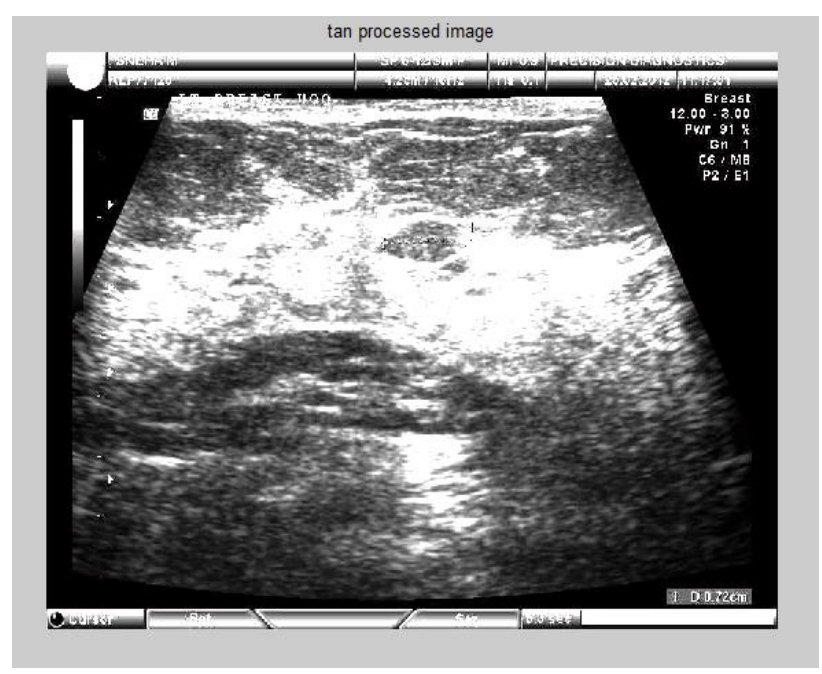

Fig.10: Tan processed image

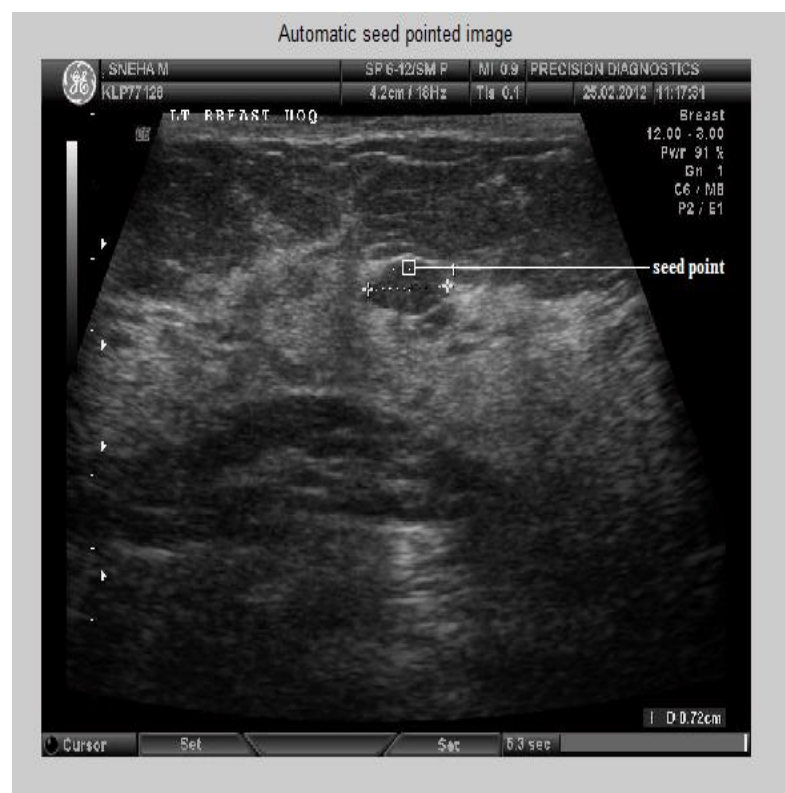

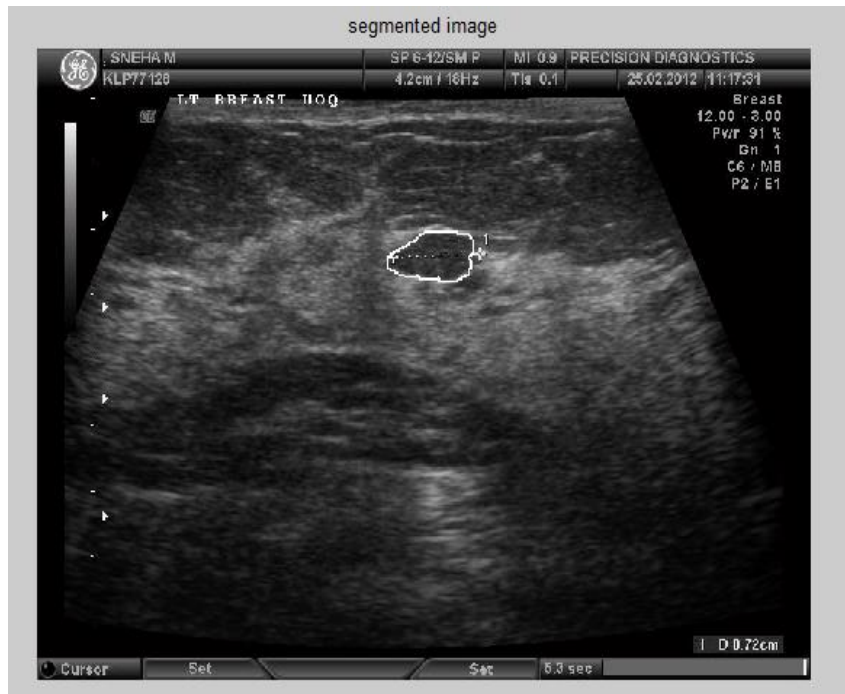

Fig.12: Automatic segmented image

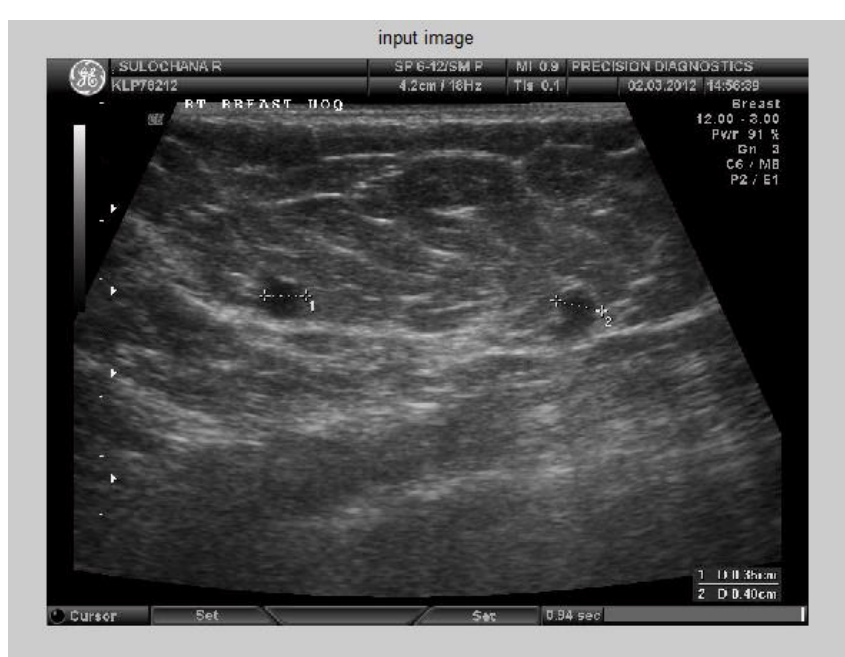

Fig.13: Input image

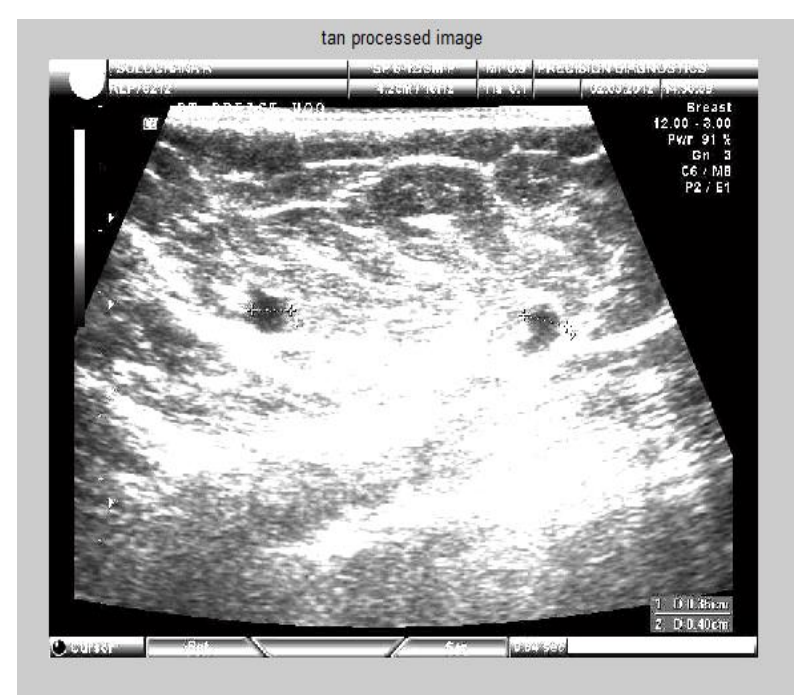

Fig.14: Tan processed image

Fig.11: Automatic seed pointed image 


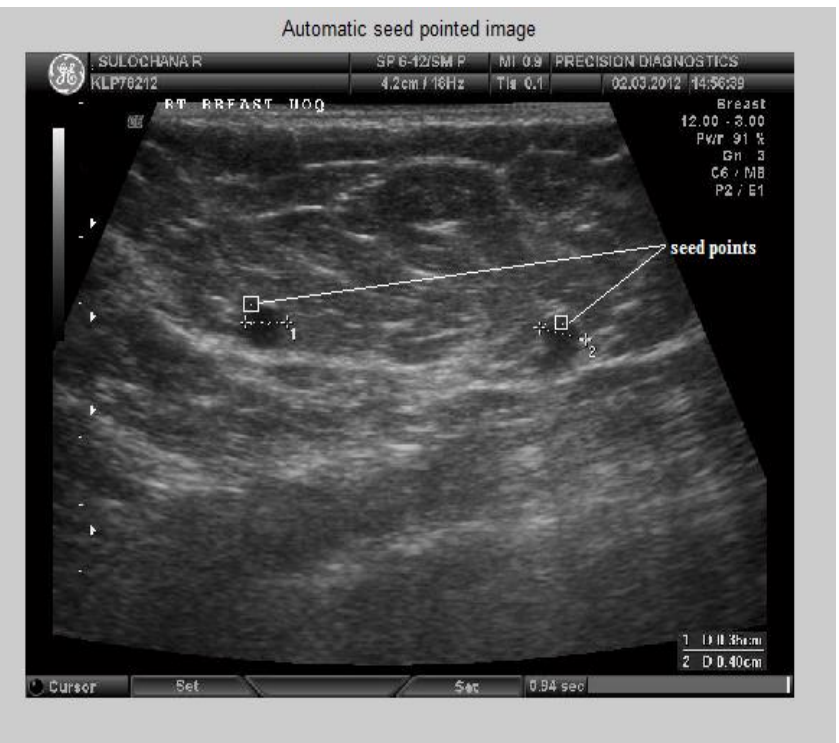

Fig.15: Automatic seed pointed image

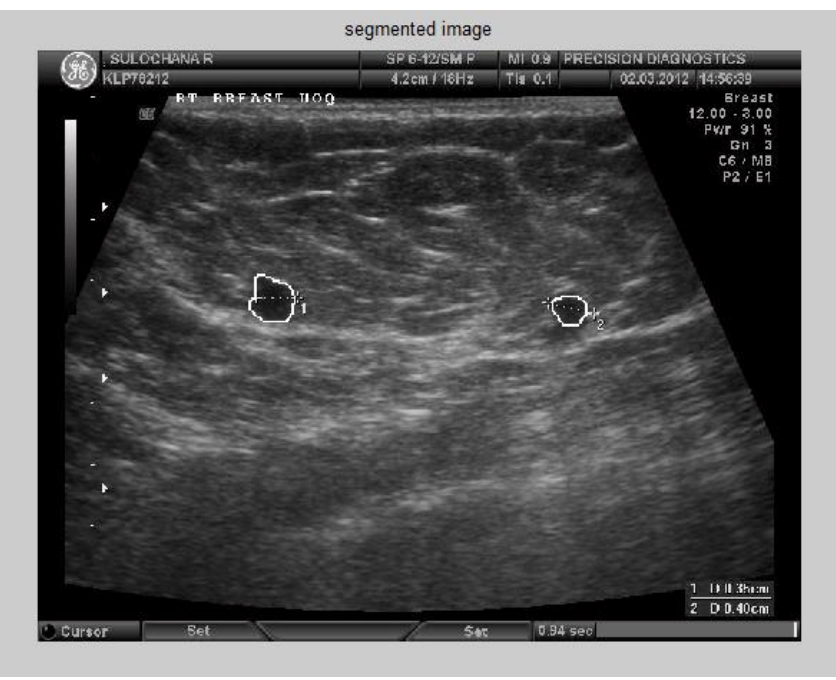

Fig.16: Automatic segmented image

Table 4: Quantitative analysis

\begin{tabular}{|c|c|c|}
\hline Specifications & No of Images & Percentage \\
\hline True Positive & 39 & $97.5 \%$ \\
\hline False Positive & 1 & $2.5 \%$ \\
\hline
\end{tabular}

The quantitative result is shown in the Table 4. If the seed point is located inside the lesion region, it is considered as true positive (TP), else it is considered as false positive (FP). Out of 40 images, the proposed method as able to automatically segment lesion in US image for 39 images. The TP rate for the database is $97.5 \%$ and the FP rate is $2.5 \%$.

\section{Conclusion}

The aim of this method is to find seed points and also segment multiple-lesion regions automatically without the manual intervention which is a difficult task. The proposed method is able to find the seed point automatically within multiple-lesion regions without the intervention of the sonographer with the TP rate of $97.5 \%$ for our database. The proposed method is robust and consumes very less time when compared to manual intervention. For efficient output of the BPN network, the network must be trained with different training images.
In this method, BPN has been used. In future, the algorithm could be trained with different neural network. Depending upon the performance of various neural networks, efficient neural network could be applied for effective outcome of the project.

\section{Acknowledgment}

The authors are grateful to Dr.Bharthi Y.Dhala, Eminent Radiologist in Precision Diagnostic, MEDALL, Chennai for providing the images and guidance.

\section{References}

[1]. Telagarapu Prabhakar, S.Poonguzhali, Automatic Detection and Classification of Benign and Malignant Lesions in Breast Ultrasound Images using Texture Morphological and Fractal Features, 978-1-5386-0882-1/17/2017 IEEE

[2]. Guita Rahbar, Angela C. Sie, Gail C. Hansen, Jeffrey S. Prince, Michelle L. Melany. Benign versus Malignant Solid Breast Masses: US Differentiation. 1999. Radiology.

[3]. Hong-Ting Chen, Yi-Ping Lien, Po-Ting Liu, Dar-Ren Chen and Yu-Len Huang. Characterization of Benign and Malignant Solid Breast Masses Using Vascular Morphology in 3D Power Doppler Ultrasound Images. 2010.3rd International Conference on Biomedical Engineering and Informatics.

[4]. Hsieh-Wei Lee, King-Chu Hung, Bin-Da Li, Sheau-Fang Lei, and Hsin-Wen Ting. Realization of high octave decomposition for breast cancer feature extraction on ultrasound images. 2011. IEEE Transactions on circuits and system. Vol 58, NO. 6.

[5]. Juan Shan, H. D., Cheng, Yuxuan Wang. A novel automatic seed point selection algorithm for breast ultrasound images. 2008 IEEE Transaction.

[6]. Neb Duric, Cuiping Li, Carri Glide-Hurst, Peter Littrup, Lianjie Huang and Jessica Lupinacc. Breast Imaging with Ultrasound Tomography: Clinical results at the Karmanos Cancer Institute. 2008 International Conference on BioMedical Engineering and Informatics.

[7]. Poonguzhali.S and Ravindran.G. A complete automatic region growing method for segmentation of masses on ultrasound images. 2006. IEEE International Conference on BioMedical and pharmaceutical Engineering.

[8]. Ruey-Feng Chang, Wen-Jie Wu, Chih-Chi Tseng, Dar-Ren Chen, and Woo Kyung Moon. 3-D snake for US in margin evaluation for malignant breast tumor excision using mammotome. 2003. IEEE transactions on Information TechnologyinBiomedicine, Vol. 25, No. 3.

[9]. Simona Moldovanu, Luminita Moraru. Mass Detection and Classification in Breast Ultrasound Image Using K-means Clustering Algorithm.2010. IEEE. Vol. 25, No. 3.

[10]. KISHORE, P.V.V., KISHORE, S.R.C. and PRASAD, M.V.D., 2013. Conglomeration of hand shapes and texture information for recognizing gestures of indian sign language using feed forward neural networks. International Journal of Engineering and Technology, 5(5), pp. 3742-3756.

[11]. RAMKIRAN, D.S., MADHAV, B.T.P., PRASANTH, A.M., HARSHA, N.S., VARDHAN, V., AVINASH, K., CHAITANYA, M.N. and NAGASAI, U.S., 2015. Novel compact asymmetrical fractal aperture Notch band antenna. Leonardo Electronic Journal of Practices and Technologies, 14(27), pp. 1-12.

[12]. KARTHIK, G.V.S., FATHIMA, S.Y., RAHMAN, M.Z.U., AHAMED, S.R. and LAY-EKUAKILLE, A., 2013. Efficient signal conditioning techniques for brain activity in remote health monitoring network. IEEE Sensors Journal, 13(9), pp. 3273-3283.

[13]. KISHORE, P.V.V., PRASAD, M.V.D., PRASAD, C.R. and RAHUL, R., 2015. 4-Camera model for sign language recognition using elliptical fourier descriptors and ANN, International Conference on Signal Processing and Communication Engineering Systems - Proceedings of SPACES 2015, in Association with IEEE 2015, pp. 34-38. 